\title{
Breast Cancer Management During the COVID-19 Pandemic: The Senologic International Society Survey
}

(1) Carole Mathelin ${ }^{1,2}$, (1) Shanti Ame $e^{1}$, (1) Stanley Anyanwu ${ }^{3}$, (1) Eli Avisar ${ }^{4}$, (D) Wahib Mohcen Boubnider ${ }^{5}$, (1) Katrin Breitling ${ }^{6}$,

(1) Hannah Ayettey Anie , (1) José Carlos Conceiçăa ${ }^{8}$, (1) Veronique Dupont ${ }^{9}$, (1) Elisabeth Elder $^{10}$, (1) Constanze Elfgen ${ }^{11}$,

(1) Tony Elonge ${ }^{12}$, (1) Edelmiro Iglesias ${ }^{13}$, (1) Shigeru Imoto ${ }^{14}$, (1) Lydia Ioannidou-Mouzaka ${ }^{15}$, (1) Elisabeth A Kappos ${ }^{16}$,

(1) Martin Kaufmann ${ }^{17}$, (1) Michael Knauer ${ }^{18}$, (1) Franck Luzuy ${ }^{19}$, (1) Marko Margaritoni2 ${ }^{20}$, (1) Mamadou Mbodj ${ }^{21}$,

(1) Alexander Mundinger ${ }^{22}$, (1) Ruben Orda ${ }^{23}$, (1) Valerijus Ostapenkoo ${ }^{24}$, (1) Serdar Özbaş², (1) Vahit Özmen ${ }^{26}$,

(1) Olivia Pagani ${ }^{27}$, (1) Tadeusz Pieńkowski ${ }^{28}$, (1) Schlomo Schneebaum ${ }^{29}$, (1) Ekaterina Shmalts ${ }^{30}$, (1) Ashraf Selim ${ }^{31}$, (1) Zotov Pavel ${ }^{32}$,

(D) Massimo Lodi' 1 , (D) Maurício Magalhales Costa ${ }^{33}$

${ }^{1}$ Institut de Cancérologie Strasbourg Europe (ICANS), Strasbourg Cedex, France

${ }^{2}$ Immediate the Senology International Society (SIS) Past President, France

${ }^{3}$ The Senology International Society Vice-President for Africa, Nigeria

${ }^{4}$ Miller School of Medicine, University of Miami, USA

${ }^{5}$ Department of Breast Surgery, Centre Pierre-et-Marie-Curie, Alger, Algeria

${ }^{6}$ Breast Center, Schaffhausen Hospital, Switzerland

${ }^{7}$ Radiotherapy Oncology and Nuclear Medicine Centre, Ghana

${ }^{8}$ Brazilian National Cancer Institute, Rio de Janeiro Federal University, Brazil

${ }^{9}$ Department of Breast Surgery, St Gallen, Switzerland

${ }^{10}$ Westmead Breast Cancer Institute, University of Sydney, Australia

${ }^{11}$ Breast Cancer Center, Zurich, Switzerland

${ }^{12}$ Hôpital Otema, Congo Democratic Republic

${ }^{13}$ The Senology International Society (SIS) Vice-President for Europe, Spain

${ }^{14}$ Kyorin University Hospital, Japan

${ }^{15}$ President of the upcoming Senology International Society (SIS) Congress, Greece

${ }^{16}$ Breast Center and Department of Plastic, Reconstructive, Aesthetic and Handsurgery, University Hospital Basel, Switzerland

${ }^{17}$ Breast Center, Bülach Hospital, Switzerland

${ }^{18}$ Breast Center Eastern Switzerland, St. Gallen, Switzerland

${ }^{19}$ Department of Breast Surgery, Hirslanden Clinic, Geneva, Switzerland

${ }^{20}$ Department of Plastic surgery, Dubrovnik General Hospital, Croatia

${ }^{21}$ Department of Nuclear Medicine, General Hospital Idrissa POUYE, Dakar, Senegal

${ }^{22}$ The Senology International Society (SIS) auditor, Germany

${ }^{23}$ The Senology International Society (SIS) International School of Senology, Israel

${ }^{24}$ National Cancer Institute Vilnius, Lithuania

${ }^{25}$ Surgical Private Practice, Ankara, Turkey

${ }^{26}$ The Senology International Society (SIS) Standing Committee, Turkey

${ }^{27}$ Department of Oncology, Breast Unit and Institute of Oncology of Southern Switzerland

${ }^{28}$ The Senology International Society (SIS) auditor, Poland

${ }^{29}$ The Senology International Society (SIS) President Elect, Israel

${ }^{30}$ Department of Oncology, Multi-field Clinical Medical Centre "Medical City", Tyumen, Russia

${ }^{31}$ Department of Radiology, Cairo University, Egypt

${ }^{32}$ Department of Oncology, Tyumen State Medical University, Russia

${ }^{33}$ The Senology International Society (SIS) President, Brazil 


\section{ABSTRACT}

Objective: In early 2020, the spread of coronavirus disease-2019 (COVID-19) led the World Health Organization to declare this disease a pandemic. Initial epidemiological data showed that patients with cancer were at high risk of developing severe forms of COVID-19. National scientific societies published recommendations modifying the patients' breast cancer (BC) management to preserve, in theory, quality oncologic care, avoiding the increased risk of contamination. The Senology International Society (SIS) decided to take an inventory of the actions taken worldwide. This study investigates COVID-19-related changes concerning BC management and analyzes the will to maintain them after the pandemic, evaluating their oncological safety consequences.

Materials and Methods: SIS network members participated in an online survey using a questionnaire (Microsoft ${ }^{\circ}$ Forms) from June $15^{\text {th }}$ to July $31^{\text {st }}$, 2020.

Results: Forty-five responses from 24 countries showed that screening programs had been suspended (68\%); magnetic resonance imagines were postponed (73\%); telemedicine was preferred when possible (71\%). Surgeries were postponed: reconstructive (77\%), for benign diseases (84\%), and in patients with significant comorbidities $(66 \%)$. Chemotherapy and radiotherapy protocols had been adapted in $28 \%$ of patients in both. Exception for telemedicine (34\%), these changes in practice should not be continued.

Conclusion: The SIS survey showed significant changes in BC's diagnosis and treatment during the first wave of the COVID-19 pandemic, but most of these changes should not be maintained. Indeed, women have fewer severe forms of COVID-19 and are less likely to die than men. The risk of dying from COVID-19 is more related to the presence of comorbidities and age than to BC. Stopping screening and delaying treatment leads to more advanced stages of BC. Only women aged over 65 with BC under treatment and comorbidities require adaptation of their cancer management.

Keywords: Breast cancer, COVID-19, SARS-CoV-2, clinical practices, survey, pandemic

Cite this article as: Mathelin C, Ame S, Anyanwu S, Avisar E, Boubnider WM, Breitling K, Anie HA, Conceição JC, Dupont V, Elder E, Elfgen C, Elonge T, Iglesias E, Imoto S, Ioannidou-Mouzaka L, Kappos EA, Kaufmann M, Knauer M, Luzuy F, Margaritoni M, Mbodj M, Mundinger A, Orda R, Ostapenko V, Özbaş S, Özmen V, Pagani O, Pieńkowski T, Schneebaum S, Shmalts E, Selim A, Pavel Z, Lodi M, Costa MM. Breast Cancer Management During the COVID-19 Pandemic: The Senologic International Society Survey. Eur J Breast Health 2021; 17(2): 188-196

\section{Key Points}

- Breast cancer diagnosis and treatment were deeply impacted since the beginning of the SARS-CoV-2 pandemic.

- In the light of recent findings on COVID-19 risk among women with breast cancer, most of these changes should not be maintained as the risk of severe COVID-19 is related to comorbidities and age rather than breast cancer.

- Only women with breast cancer aged over 65 or with comorbidities require adaptation of their cancer management, according to the Senologic International Society.

\section{Introduction}

At the end of 2019, a new coronavirus (SARS-CoV-2) caused pneumonia in several patients epidemiologically linked to a Wuhan market (Hubei province, China). In early 2020, the spread of coronavirus disease-2019 (COVID-19) led the World Health Organization to declare this disease a pandemic. Despite extraordinary measures implemented in many countries, the epidemic spread, with mortality significantly higher than influenza. Initial epidemiological data showed that patients who were older and/or had comorbidities, and notably cancer, were at higher risk of developing severe forms of COVID-19 $(1,2)$. As breast cancer (BC) is the leading cancer among women worldwide, with more than 2 million new cases and more than 650,000 deaths each year (3), the situation was of particular concern for women with BC. Emergency health actions have been implemented in various countries, involving changes in the treatment and care circuits for numerous cancers, including BC. Many national scientific societies published a series of recommendations modifying $\mathrm{BC}$ patient management to preserve, in theory, quality oncologic care while avoiding an increased risk of contamination by SARS-CoV-2 (46). In addition, postponing BC surgeries freed equipment and health care staff to support COVID-19 patient care.

The Senologic International Society (SIS) has always been dedicated to promoting breast health and improving $\mathrm{BC}$ patients' care, considering medical, social, economic, and ethical constraints. In this pandemic context, the SIS, by its active members, experts, and its global network, decided to assess the actions taken worldwide, the clinical practice changes, and the particularities observed in different countries regarding BC management. The SIS survey's first objective was to investigate and share COVID-19-related changes in clinical practices concerning $\mathrm{BC}$ management since the beginning of the pandemic. The second objective was to analyze the will to maintain these changes later in treatment modalities and care pathways, considering the consequences of these oncological safety changes.

\section{Materials and Methods}

SIS network members were invited to participate in an online survey using the Microsoft Forms questionnaire. Between June $15^{\text {th }}$ and July $31^{\text {st }}, 2020$, participants were invited to answer the questionnaire via e-mail. The answers were directly recorded into an online database, and only one response per participant was allowed. However, more than one response was authorized for each country in case of national disparities.

The online survey consisted of 17 questions. Question 1 was about the participant's origin. The number of deaths due to Coronavirus recorded in his/her country at the moment of the survey, and question 17 was about the participant's profile. Next, we questioned the participants about $\mathrm{BC}$ screening programs, radiological practices (question 4), pathological analyses (question 5), telemedicine (question 6), secondary reconstruction surgeries (question 7), benign lesion surgeries (question 8), surgery for patients with 
comorbidities (question 9), chemotherapy practices (questions 10 and 11), radiotherapy practices (question 12 and 13), and clinical trials (question 14). We then asked about the management after the lockdown period, if applicable (question 15), and the practice changes that should be maintained thereafter (question 16). The questionnaire is available as Supplementary Material S1 in Appendix 1 .

\section{Results}

We received 45 completed questionnaires in total. Participants came from 24 countries on six continents (Figure 1): Algeria, Australia, Brazil, Democratic Republic of Congo, Croatia, Ecuador, Egypt, France, Germany, Ghana, Greece, Israel, Jamaican Republic, Japan, Lithuania, Nigeria, Poland, Romania, Russia, Senegal, Spain, Switzerland, Turkey, and the United States. The survey results are reported in Table 1 .

In our survey, $38 \%$ of participants were oncological surgeons, $4 \%$ medical oncologists, $4 \%$ radiotherapists, $4 \%$ radiologists, $2 \%$ nuclear physicians, and $2 \%$ anesthesiologists. The other $46 \%$ had more than one specialty (a radiologist and nuclear physician or radiotherapist and medical oncologist). Fifty-six percent of respondents had a public or governmental practice, $13 \%$ a practice in the private sector, and 31\% were involved with both activities. No difference was noted between the public and private sectors regarding $\mathrm{BC}$ management.

\section{Diagnosis and consultations}

Most participants (69\%) reported that organized or individual screening programs were suspended during the pandemic. Sixteen percent of participants reported that screening programs were maintained (Japan, Senegal, Switzerland-in one canton, Jamaican Republic, Croatia, and Algeria). No response was available in the other cases, as these countries have no screening programs.

Participants answered that breast magnetic resonance imaging (MRI) was more often maintained (73\%). In contrast, it was suspended for $22 \%$ of participants (Switzerland in three cantons, France, Democratic Republic of Congo, Nigeria, Ecuador, and Turkey) due to the difficulty of adequately disinfecting the devices. No response was available in the other cases.

Concerning teleconsultations and phone consultations, most participants $(71 \%)$ preferred this solution, when possible, during the pandemic. On the other hand, $24 \%$ of participants preferred classical consultations. No response was available in the other cases.

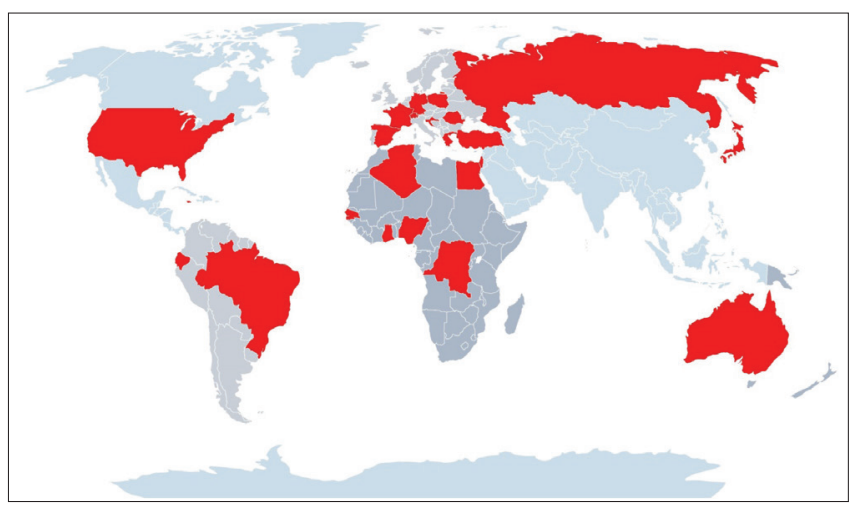

Figure 1. World map with countries that participated in this survey
Table 1. Survey results

Question/Answers

n

(\%)

Are/were organized or individual screening programs suspended in your country/state/province during the pandemic?

Yes 31

No

Not applicable/no opinion

7

Are/were breast MRIs performed during the pandemic?

Yes

33

No

10

Not applicable/no opinion

2

Are/were extemporaneous examinations avoided during the pandemic?

Yes

30

No

Not applicable/no opinion

14

Whenever possible, are/were teleconsultations or telephone consultations preferred during the pandemic?

Yes

No

Not applicable/no opinion

Are/were secondary reconstruction surgeries postponed during the pandemic?

Yes

No

35

Not applicable/no opinion

5

Are/were surgeries involving benign lesions postponed during the pandemic?

Yes

38

No

5

Not applicable/no opinion

2

For patients with significant comorbidities making the risk of complications high in case of COVID-19 (elderly subjects, chronic respiratory or cardiac pathology, immunosuppression) is/was the surgical procedure postponed?

Yes

No

30

Not applicable/no opinion

Are/were chemotherapy protocols modified to reduce the hospital stay?

Yes

13

No

Not applicable/no opinion

22 
Table 1. Continued

\section{Question/Answers}

n

If yes, how?*

$\begin{array}{lcc}\text { Outpatient treatments } & 4 & (9) \\ \text { Postponed } & 4 & (9) \\ \text { Protocol modification } & 8 & (17) \\ \text { Treatment order modification } & 1 & (2) \\ \text { No/no opinion } & 31 & (69) \\ \text { Are/were radiotherapy protocols modified to reduce the } \\ \text { hospital stay? } & 13 \\ \text { Yes } & 20 \\ \text { No } & 12 & (29) \\ \text { Not applicable/no opinion } & & (44) \\ \text { If yes, how?* } & 1 & (2) \\ \text { Outpatient treatments } & 2 & (4) \\ \text { Postponed } & 8 \\ \text { Protocol modification } & 2 & (18) \\ \text { Treatment order modification } & 32 \\ \text { No/no opinion } & 10 \\ \text { Are/were patients with both breast cancer and covID-19 } \\ \text { infection excluded from the COVID-19 trials? } \\ \text { Yes } & 4 & (71) \\ \text { No } & 31 & (69) \\ \text { Not applicable/no opinion } & & \end{array}$

After the period of deconfinement, will you (or have you) anticipate(d) and prioritize(d) the management of patients with breast pathologies not treated during the pandemic?

Yes

31

No

3

11

Not applicable/no opinion

Are/were some practice changes beneficial and should they be maintained thereafter? Which changes?*

Telemedicine implementation

Outpatient treatments

Neoadjuvant endocrine therapy

Public-Private health system

collaboration

Improved symptomatic treatment

SARS-CoV-2 screening before

treatment

Improved hygiene measures /

Personal Protective Equipment

Improved personalized treatments

Less extemporaneous examinations

No/no opinion

1

2

1

2

3

2

17

4)

(1)

9)

(6)

)

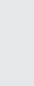
$(8 \%)$, treatment postponement $(15 \%)$ and/or treatment order
modification $(15 \%)$.

\section{Clinical trials}

Four participants (9\%) mentioned that patients with BC and COVID-19 were excluded from clinical trials concerning COVID-19. Ten participants responded negatively (22\%). This question was not applicable, or the participant had no opinion in $69 \%$ of cases.

\section{After the lockdown period}

Most participants (69\%) anticipated and prioritized the management of patients with breast pathologies not treated during the pandemic. We observed that $24 \%$ of participants did not respond, as, in some countries, there was no lockdown at the time of the survey.

Thirty-six percent of participants found that telemedicine implementation should be maintained after the pandemic, for example, for $\mathrm{BC}$ survivors. The remaining practice changes should not be maintained to avoid the pejorative evolution of some patients.

\section{Mortality}

Our survey did not provide any results regarding mortality among BC patients, as it concerned practice changes and was not an observational study. 


\section{Discussion and Conclusion}

The SIS conducted an international survey to investigate and share COVID-19 related changes in clinical practices concerning BC management during the pandemic.

\section{Strengths and limitations of the survey}

Participants were from 24 countries, as shown in Figure 1. These countries represented 1.701 billion people and $22 \%$ of the world's population. Participants had different disciplines and covered most breast specialties: surgery (general and gynecological), medical oncology, radiotherapy, radiology, nuclear medicine, and anesthesiology. In addition, they were from different types of practices, working in both university hospitals and private practices. In some cases, practices were heterogeneous among healthcare providers within each country, especially if the pandemic affected their regions differently. However, the SIS survey showed that many countries had adopted similar measures. Concerning diagnosis, screening programs had often been suspended, and MRI use was reduced. Therapeutically, non-urgent surgery had been postponed, whereas medical and radiotherapy treatments had been adopted.

All these changes were based on initial epidemiological data hypothesizing that patients with cancer were at a higher risk of developing severe forms of COVID-19. For example, at the beginning of the pandemic, a study published by Liang et al. (1) showed that patients with cancer had a higher risk of developing a severe form of COVID-19, including invasive ventilation, intensive care unit admissions, or death [odds ratio (OR): 5.34, 95\% confidence interval (CI): 1.80-16.18; $\mathrm{p}=0.0026]$. However, even when the study included 1,590 patients, only $18(1 \%)$ had a history of cancer, and among them, BC was present in only three patients. Similarly, Fu et al. (7) showed that cancer patients, in general, were at a higher risk of being infected with SARS-CoV-2 and had higher mortality rates. Likewise, de Azambuja et al. (8) showed that in a cohort of 13,594 patients (of whom 1,187 had solid cancers), cancer was associated with higher COVID-19 related mortality. However, in April 2020, Miyashita et al. (2) published a report on 5,688 patients, including 344 with cancer, of whom 57 had BC. They found that patients with cancer were at significantly higher risk of requiring invasive ventilation (relative risk: 1.89; 95\% CI: $1.37-2.61$ ) but not of death (relative risk: $1.15 ; 95 \%$ CI: $0.84-1.57$ ) except for patients aged up to 50 years old (relative risk: 5.01; 95\% CI: 1.55-16.2). Unfortunately, no subgroup analysis based on the cancer type was provided.

Since patients with cancer might be at a higher risk in COVID-19, several scientific societies published guidelines to adapt BC management during the pandemic. Worldwide, $\mathrm{BC}$ care pathways were disrupted $(9,10)$ : screening was discontinued, surgeries were delayed, chemotherapy and radiotherapy protocols were adapted to reduce hospital stays or the number of sessions. BC treatments were modified since the beginning of the pandemic. First, chemotherapy protocols were changed to reduce hospital stays. For instance, taxane-based protocols with weekly administrations (i.e., paclitaxel) were replaced by docetaxel administrations every three weeks. Otherwise, some other protocols were discontinued or postponed. In some metastatic situations, chemotherapy regimens were changed to an oral treatment such as capecitabine. The main goal was to decrease potential exposure to SARS-CoV-2 and complications in concomitant chemotherapy and COVID-19. Similarly, an American team published results that adjuvant and neoadjuvant chemotherapy were modified in $41 \%$ of cases to reduce the risk of SARS-CoV-2 infection (11). In contrast, oral treatments were modified in only $15 \%$ of cases (11).

Radiotherapy was also changed during the pandemic. Hypofractionated regimens were preferred for BC treatment, whereas in other cases, treatment was discontinued. Spencer et al. (12) published a report on radiotherapy modifications in the United Kingdom during the pandemic and found similar results. For instance, treatment with ultrahypofractionated regimens (26 Gy in 5 fractions) greatly increased from 0.2\% in April 2019 to 60.6\% in April 2020.

Finally, surgery was also impacted. As reported in the survey, benign and reconstructive surgeries were postponed. In fragile patients, cancer surgeries have also been postponed, and in some cases, neoadjuvant endocrine therapy was introduced. As reported in a survey in the United States published by Park et al. (13), most oncologists (medical, radiotherapists, and surgeons) changed their BC management, and neoadjuvant endocrine therapies went from rarely to frequently prescribed during the pandemic. In addition, most participants found it reasonable to delay surgery without the use of endocrine therapy for 1-2 months, but not for three months (13).

Moreover, during the pandemic, face masks were of paramount importance for COVID-19 prevention, and they were mandatory in healthcare structures in many countries. Clearly, since the beginning of the pandemic, the daily wearing of face masks had been another important change in worldwide practices.

So, important measures were taken at the beginning of the pandemic, even if they were based on preliminary data. Retrospectively, and based on the impact of cancer on the evolution of COVID-19, some proceedings were abandoned. The SIS survey showed that, apart from the development of telemedicine and the wearing of face masks, all these changes would not be definitively implemented by breast specialists' members of the SIS for the following reasons.

\section{Particularities of COVID-19 in women currently treated for BC}

First, women have a lower risk of having COVID-19 than men. Growing evidence in the scientific literature shows that men are at higher risk of severe forms of COVID-19 and have higher mortality (14-16). These findings are true for most countries, except for Canada, Vietnam, and Belgium (17), where these differences can be explained by epidemiological factors such as age $(15,16)$, health behavior $(15)$, socioeconomic context (15), different comorbidities (16), and different immune responses (18). Consequently, data concerning women with cancer was probably too alarming.

Second, women with BC have a lower risk of having COVID-19 than women with other cancers. It was supposed that SARS$\mathrm{CoV}-2$ infection could have higher direct mortality in BC patients, as they may develop more severe forms of the disease. However, new evidence was published that softened the initial fear. Indeed, COVID-19's impact on mortality is not the same according to cancer type. By analyzing the cancer type, Lee et al. (19) found in a British cohort of 800 patients that $\mathrm{BC}$ was at low risk of mortality compared with other cancers (OR: $0.48(0.28-0.84), p=0.009)$. In addition, the authors found that COVID-19-related mortality was principally caused by age, gender, and comorbidities. Vuagnat et al. (20) published a study on 76 patients with early or metastatic BC and suspected COVID-19 among a French cohort of 15,600 patients. They found that $10 \%$ were transferred to an intensive care unit, and 
$7 \%$ died (all of whom had significant non-cancer comorbidities). The statistical analysis found that hypertension and age $(>70)$ were the two factors associated with a higher risk of intensive care unit admission and/or death. Interestingly, a history of radiotherapy or current oncological treatment was not associated with mortality. Kalinsky et al. (21) published a report on 27 patients with BC in a cohort of 4,515 patients with COVID-19 from New York City. They found a mortality rate of $4 \%(n=1)$, an 87 -year-old male with coronary artery disease, hypertension, and smoking history. De Melo et al. (22) analyzed a population of 40 patients with BC in a Brazilian cohort of 181 patients with cancer and COVID-19. They found that mortality was associated with symptomatic COVID-19 and the presence of two or more metastatic sites in the multivariate analysis. According to the literature, COVID-19's mortality in BC patients seems to be lower than initially estimated compared with other cancers.

Third, late diagnosis because of the lockdown and screening discontinuation may lead to increased BC-related mortality. Vanni et al. (23) conducted a multicentric cohort study in Italy to evaluate the impact of screening suspension and surgical delay on BC staging. They included 432 patients and found that the disease was more advanced at diagnosis with more lymph node involvement. In addition, Maringe et al. (24) studied the impact of COVID-19 and the lockdown period in England and established a predictive model of BC mortality. They estimated that there would be a $7.9 \%-9.6 \%$ increase in the number of deaths due to BC up to year five after diagnosis in England. Johnson et al. (25) conducted a meta-analysis to evaluate how surgical delay affected survival in breast, lung, and colon cancers. They included 25 articles, of which 12 concerned BC. They found that delaying surgery for 12 weeks might decrease overall BC survival (hazard ratio: 1.46, 95\% CI: 1.28-1.65). When BCs were analyzed by stage, the authors found that survival was decreased in early stages (I and II, respectively 1.27, 95\% CI: 1.16-1.40; and II 1.13, 95\% CI: 1.02-1.24) but not in advanced BC.

Considering these findings, many $\mathrm{BC}$ specialists suggest that young and middle-aged patients with BC and without comorbidities should be treated without delay during the pandemic. Only patients aged 65 years old or older and with one or more comorbidities (hypertension, diabetes, chronic respiratory disease, and obesity) should have their treatment adapted to lower COVID-19 risk.

\section{Should caution be maintained for patients formerly treated for BC?}

Women treated over five years ago for $\mathrm{BC}$ are not at increased risk of having the severe form of COVID-19. However, the effects of COVID-19 on BC recurrence were hypothesized. For example, Francescangeli et al. (26) suggested that potential mechanisms could be implicated in reawakening dormant BC cells. In particular, pulmonary dormant $\mathrm{BC}$ cells could be reactivated by COVID-19 infection via immune pathways. In a review published by Silvin et al. (27), the authors highlight that severe COVID-19 is linked to an inflammatory burst and lymphopenia related to carcinogenesis and may aggravate cancer prognosis. Even if data are published on COVID-19 immunemediated cancer recurrence, further studies are needed to assess this potential risk. When possible, telemedicine should be encouraged for $\mathrm{BC}$ survivors' follow-up to avoid contact with SARS-CoV-2.

In conclusion, the SIS survey showed significant changes in $\mathrm{BC}$ diagnosis and treatment changes during the first wave of the COVID-19 pandemic. Participants in this survey emphasized that most of these changes should not be maintained. Indeed, women have less severe forms of COVID-19 and are less likely to die from COVID-19 than men. The risk of dying from COVID-19 is more related to the presence of comorbidities and age than to BC. Suspending screening and delaying cancer treatment led to more advanced stages of $\mathrm{BC}$. All these delays in $\mathrm{BC}$ management may potentially influence $\mathrm{BC}$ mortality, even if this effect will not become apparent before probably 7-10 years. Only women aged over 65 with cancer under treatment and comorbidities require an adaptation of their cancer management. When possible, telemedicine should be encouraged for BC survivors' follow-up.

\section{Acknowledgements}

We thank Gérard Hrodej for the help to contact the SIS network members.

Ethics Committee Approval: Please find attached the positive advice, reference CE-2021-38, of our Ethics Committee "Comité d'Ethique des Facultés de Médecine, d'Odontologie, de Pharmacie, des Ecoles d'Infirmières, de Kinésithérapie, de Maïeutique et des Hôpitaux» of March 22 2021.

Informed Consent: Retrospective study.

Peer-review: Externally peer-reviewed.

\section{Authorship Contributions}

Conception: C.M., S.A., M.L., M.M.C.; Design: C.M., M.L., M.M.C.; Data Collection or Processing: C.M., St.A., E.A., W.M.B., K.B., H.A.A., J.C.C., V.D., E.E., C.E., T.E., E.I., S.I., L.I.M., E.A.K., M.K., Mi.K., F.L., M.M., Ma.M., A.M., R.O., V.O., S.Ö., V.Ö., O.P., T.P., S.S., E.S., Z.P., M.L., M.M.C.; Analysis or Interpretation: C.M., St.A., E.A., W.M.B., K.B., H.A.A., J.C.C., V.D., E.E., C.E., T.E., E.I., S.I., L.I.M., E.A.K., M.K., Mi.K., F.L., M.M., Ma.M., A.M., R.O., V.O., S.Ö., V.Ö., O.P., T.P., S.S., E.S., Z.P., M.L., M.M.C.; Literature Search: C.M., S.A., M.L., M.M.C.; Writing: C.M., S.A., M.L., M.M.C.

Conflict of Interest: No conflict of interest was declared by the authors.

Financial Disclosure: The authors declared that this study received no financial support.

\section{References}

1. Liang W, Guan W, Chen R, Wang W, Li J, Xu K, et al. Cancer patients in SARS-CoV-2 infection: a nationwide analysis in China. Lancet Oncol 2020; 21: 335-337. (PMID: 32066541) [Crossref]

2. Miyashita H, Mikami T, Chopra N, Yamada T, Chernyavsky S, Rizk D, et al. Do patients with cancer have a poorer prognosis of COVID-19? An experience in New York City. Ann Oncol 2020; 31: 1088-1089. (PMID: 32330541) [Crossref]

3. Bray F, Ferlay J, Soerjomataram I, Siegel RL, Torre LA, Jemal A. Global cancer statistics 2018: GLOBOCAN estimates of incidence and mortality worldwide for 36 cancers in 185 countries. CA Cancer J Clin 2018; 68 : 394-424. (PMID: 30207593) [Crossref]

4. Darai E, Mathelin C, Gligorov J. Breast cancer management during the COVID 19 pandemic: French guidelines. Eur J Breast Health 2020; 16: 160-161. (PMID: 32656513) [Crossref]

5. Soran A, Gimbel M, Diego E. Breast cancer diagnosis, treatment and follow-up during COVID-19 pandemic. Eur J Breast Health 2020; 16: 86-88. (PMID: 32285027) [Crossref]

6. Cakmak GK, Ozmen V. Sars-CoV-2 (COVID-19) outbreak and breast cancer surgery in Turkey. Eur J Breast Health 2020; 16: 83-85. (PMID: 32285026) [Crossref] 
7. Fu L, Wang B, Yuan T, Chen X, Ao Y, Fitzpatrick T, et al. Clinical characteristics of coronavirus disease 2019 (COVID-19) in China: a systematic review and meta-analysis. J Infect 2020; 80: 656-665. (PMID: 32283155) [Crossref]

8. de Azambuja E, Brandão M, Wildiers H, Laenen A, Aspeslagh S, Fontaine C, et al. Impact of solid cancer on in-hospital mortality overall and among different subgroups of patients with COVID-19: a nationwide, population-based analysis. ESMO Open 2020; 5: e000947. doi: 10.1136/ esmoopen-2020-000947. (PMID: 32978251) [Crossref]

9. de Azambuja E, Trapani D, Loibl S, Delaloge S, Senkus E, Criscitiello $\mathrm{C}$, et al. ESMO Management and treatment adapted recommendations in the COVID-19 era: breast cancer. ESMO Open 2020; 5(Suppl 3): e000793. doi: 10.1136/esmoopen-2020-000793. (PMID: 32439716) [Crossref]

10. Seely JM, Scaranelo AM, Yong-Hing C, Appavoo S, Flegg C, Kulkarni S, et al. COVID-19: safe guidelines for breast imaging during the pandemic. Can Assoc Radiol J 2020; 71: 459-469. (PMID: 32452700) [Crossref]

11. Lin DD, Meghal T, Murthy P, Mo L, D'Silva A, Huang Y, et al. Chemotherapy treatment modifications during the COVID-19 outbreak at a Community Cancer Center in New York City. JCO Glob Oncol 2020: 1298-1305. (PMID: 32795196) [Crossref]

12. Spencer K, Jones CM, Girdler R, Roe C, Sharpe M, Lawton S, et al. The impact of the COVID-19 pandemic on radiotherapy services in England, UK: a population-based study. Lancet Oncol 2021; 22: 309-320. (PMID: 33493433) [Crossref]

13. Park KU, Gregory ME, Lustberg MB, Bazan JG, Shen C, Rosenberg SM, et al. Abstract SS2-05: emerging from COVID-19 pandemic: provider perspective on use of neoadjuvant endocrine therapy (NET) in early stage hormone receptor positive breast cancer. Cancer Res 2021; 81(Suppl 4): SS2-05-SS2-. [Crossref]

14. Chen N, Zhou M, Dong X, Qu J, Gong F, Han Y, et al. Epidemiological and clinical characteristics of 99 cases of 2019 novel coronavirus pneumonia in Wuhan, China: a descriptive study. Lancet 2020; 395: $507-$ 513. (PMID: 32007143) [Crossref]

15. Gebhard C, Regitz-Zagrosek V, Neuhauser HK, Morgan R, Klein SL. Impact of sex and gender on COVID-19 outcomes in Europe. Biol Sex Differ 2020; 11: 29. (PMID: 32450906 ) [Crossref]

16. Meng Y, Wu P, Lu W, Liu K, Ma K, Huang L, et al. Sex-specific clinical characteristics and prognosis of coronavirus disease-19 infection in Wuhan, China: a retrospective study of 168 severe patients. PLoS Pathog 2020; 16:e1008520. doi: 10.1371/journal.ppat.1008520. (PMID: 3234374) [Crossref]
17. Global Health 5050. The Covid-19 sex-disaggredated data tracker. Available from: https://globalhealth5050.org/2020 [Crossref]

18. Takahashi T, Ellingson MK, Wong P, Israelow B, Lucas C, Klein J, et al. Sex differences in immune responses that underlie COVID-19 disease outcomes. Nature 2020; 588: 315-320. (PMID: 32846427) [Crossref]

19. Lee LY, Cazier JB, Angelis V, Arnold R, Bisht V, Campton NA, et al. COVID-19 mortality in patients with cancer on chemotherapy or other anticancer treatments: a prospective cohort study. Lancet 2020; 395: 1919-1926. (PMID: 3247368) [Crossref]

20. Vuagnat P, Frelaut M, Ramtohul T, Basse C, Diakite S, Noret A, et al. COVID-19 in breast cancer patients: a cohort at the Institut Curie hospitals in the Paris area. Breast Cancer Res 2020; 22: 55. (PMID: 32460829) [Crossref]

21. Kalinsky K, Accordino MK, Hosi K, Hawley JE, Trivedi MS, Crew $\mathrm{KD}$, et al. Characteristics and outcomes of patients with breast cancer diagnosed with SARS-Cov-2 infection at an academic center in New York City. Breast Cancer Res Treat 2020; 182: 239-242. (PMID: 32405915) [Crossref]

22. de Melo AC, Thuler LCS, da Silva JL, de Albuquerque LZ, Pecego AC, Rodrigues LOR, et al. Cancer inpatients with COVID-19: a report from the Brazilian National Cancer Institute. PLoS One 2020; 15: e0241261. doi: 10.1371/journal.pone.0241261. (PMID: 33104715) [Crossref]

23. Vanni G, Tazzioli G, Pellicciaro M, Materazzo M, Paolo O, Cattadori F, et al. Delay in breast cancer treatments during the first COVID-19 lockdown. a multicentric analysis of 432 Patients. Anticancer Res 2020; 40: 7119-7125. (PMID: 33288611) [Crossref]

24. Maringe C, Spicer J, Morris M, Purushotham A, Nolte E, Sullivan R, et al. The impact of the COVID-19 pandemic on cancer deaths due to delays in diagnosis in England, UK: a national, population-based, modelling study. Lancet Oncol 2020; 21: 1023-1034. (PMID: 32702310) [Crossref]

25. Johnson BA, Waddimba AC, Ogola GO, Fleshman JW, Jr., Preskitt JT. A systematic review and meta-analysis of surgery delays and survival in breast, lung and colon cancers: implication for surgical triage during the COVID-19 pandemic. Am J Surg 2020; S0002-9610(20)30793-5. doi: 10.1016/j.amjsurg.2020.12.015. (PMID: 33317814) [Crossref]

26. Francescangeli F, De Angelis ML, Zeuner A. COVID-19: a potential driver of immune-mediated breast cancer recurrence? Breast Cancer Res 2020; 22: 117. (PMID: 33126915) [Crossref]

27. Silvin A, Chapuis N, Dunsmore G, Goubet AG, Dubuisson A, Derosa $\mathrm{L}$, et al. Elevated calprotectin and abnormal myeloid cell subsets discriminate severe from mild COVID-19. Cell 2020; 182: 1401-18 e18. doi: 10.1016/j.cell.2020.08.002. (PMID: 32810439) [Crossref] 
Appendix 1. Supplementary material

\section{Breast cancer and COVID-2019}

Breast cancer is a major public health problem in the world and leading cause of cancer deaths in women. The spread of COVID-19 led many scientific societies to publish a series of recommendations modifying the management of breast cancer patients during the COVID-19 pandemic. The 2 objectives of these recommendations were to ensure quality oncology care for breast cancer patients while avoiding increasing their risk of contamination by COVID-19.

The international Society of Senology (SIS) is dedicated to promoting breast health and improving the care of breast cancer patients, taking into consideration, medical, social, economic and ethical constraints. In the context of the COVID-19 pandemic, the SIS, by its active members, experts and global Network must share the actions taken worldwide and the changes in practices observed in different countries and the particularities of breast cancer management during the period of deconfinement.

Deadline for response : $30^{\text {th }}$ of June 2020

*Obligatoire

\section{From which country / state or province are you from?*}

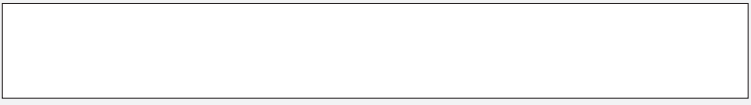

2. Do you know how many deaths due to SARS-CoV-2 are recorded in your country / state /province? (numerical response)

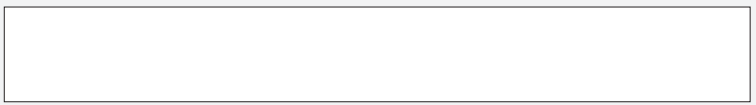

La valeur doit être un nombre

3. Are/Were organized or individual screening programs suspended in your country / state / province during the pandemic?*

Yes

No

No individual or organized screening program exists in my country

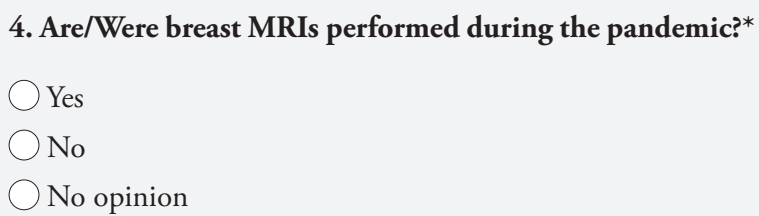

\section{Are/Were extemporaneous examinations avoided during} the pandemic?*

Yes

No

No opinion
6. Whenever possible, are/were teleconsultations or telephone consultations preferred during the pandemic?*

Yes

No

No opinion

7. Are/Were secondary reconstruction surgeries postponed during the pandemic?*

Yes

No

No opinion

8. Are/Were surgeries involving benign lesions postponed during the pandemic?*

Yes
No
No opinion

9. For patients with significant co-morbidities making the risk of complications high in case of COVID-19 (elderly subjects, chronic respiratory or cardiac pathology, immunosuppression...) is/was the surgical procedure postponed?*

Yes

No

No opinion

10. Are/Were chemotherapy protocols modified to reduce the hospital stay?*
Yes
No
No opinion 
11. If yes, how?

12. Are/Were radiotherapy protocols modified to reduce the hospital stay?*

Yes
No
No opinion

13. If yes, how?

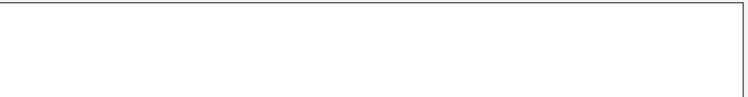

14. Are/Were patients with both breast cancer and COVID-19 infection excluded from the COVID-19 trials?*

Yes
No
No opinion

15. After the period of deconfinement, will you (or have you) anticipate(d) and prioritize(d) the management of patients with breast pathologies not treated during the pandemic?*

Yes

No

No opinion or not applicable
16. Are/Were some practice changes beneficial and should they be maintained thereafter? Which changes?*

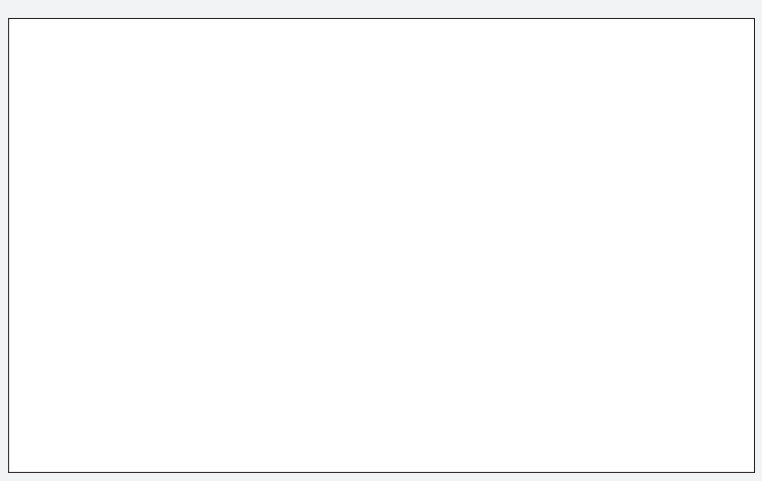

17. If you wish to participate to the publication of this survey in the European Journal of Breast Health, please state below your First name, Last name, email, affiliations (and ORCiD number if you have one)

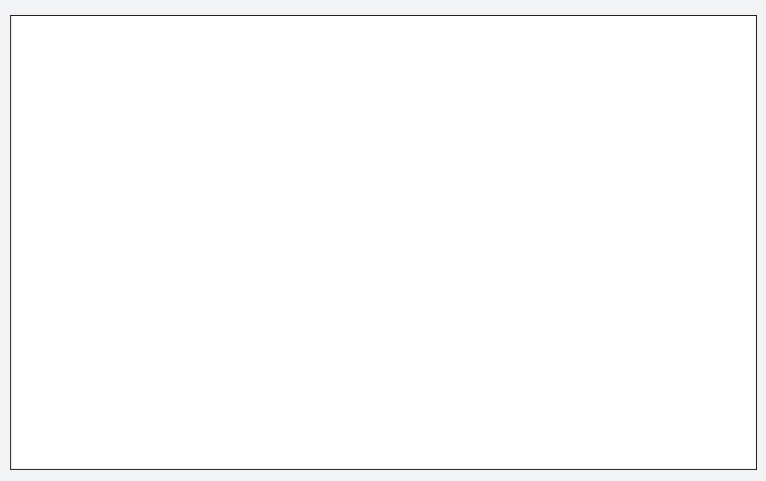

